\title{
Special issue on real-time computer vision in smart cities
}

\author{
Antonio S. Montemayor · Juan J. Pantrigo • \\ Luis Salgado
}

Published online: 24 July 2014

(c) Springer-Verlag Berlin Heidelberg 2014

According to United Nations, more than half of the population of the Earth now lives in urban areas. The need to rethink the city in efficient and modern ways, including new actors in the scene such as automatic decision makers, has motivated plenty of national and international initiatives to foster ICT research and innovation in this field. For the European Union, the sustainable development of urban areas is a challenge of key importance: it requires new, efficient, and user-friendly technologies and services, thus becoming one of the main topics within the European Research Programme "Horizon 2020".

The smart city concept is defined as an urban area that creates sustainable economic development and high quality of life by excelling in six key areas: economy, mobility, environment, people, living, and government. Small changes may have big impact on these areas, improving the well-being of its citizens, the quality of its services and reinforcing prevention. Reaching excellence in these key areas can be achieved through strong human capital, social capital, and/or ICT infrastructure. Using smart devices and

\footnotetext{
A. S. Montemayor $(\bowtie) \cdot$ J. J. Pantrigo

Department of Computer Science, Universidad Rey Juan Carlos, 28933 Móstoles, Madrid, Spain

e-mail: antonio.sanz@urjc.es

J. J. Pantrigo

e-mail: juanjose.pantrigo@urjc.es

L. Salgado

Video Processing and Understanding Laboratory, Universidad Autónoma de Madrid, 28049 Madrid, Spain

e-mail: L.salgado@gti.ssr.upm.es

L. Salgado

Grupo de Tratamiento de Imágenes, Universidad Politécnica de Madrid, 28040 Madrid, Spain
}

agents will enrich city infrastructures and computer vision may have an important role in some contexts.

The computer vision field is nowadays mature enough to demonstrate its possibilities beyond the surveillance and law enforcement purposes. It is true that its first use has been mainly motivated by security reasons, but new proposals are gaining attention in the society. From the autonomous car idea to the automatic interpretation of people interaction, groundbreaking applications open new ways to contribute to goals of this smart city concept.

This special issue includes six contributions to the development of smart cities in the computer vision context. Selected from twenty-two submitted papers after a multiround review process, they make substantial contributions in the use and/or development of real-time computer-visionbased systems in the areas of intelligent transportation systems (ITS), human activity recognition, and sustainable energy.

The paper by Vélez et al. presents "A reconfigurable embedded vision system for advanced driver assistance". This paper describes an advanced driver assistance system implemented by a system on chip composed of a programmable logic that supports parallel processing necessary for a fast pixel-level analysis, and a microprocessor suited for serial decision making. The authors develop a lane departure warning system and compare the obtained results with related works in the literature.

Salvadori et al. entitled their work as "A Low Cost Vehicles-Counter for Next Generation ITS". It describes a prototype based on line sensor cameras as a low-cost vehicle counter solution. As this application needs from a high speed acquisition, the entire pipeline is a low-complexity design for an embedded device but offers comparable results to the state of the art. Its experimental results are validated using an embedded hardware platform with very limited computational resources. 
The paper by De-Maeztu et al. "A temporally consistent grid-based visual odometry framework for multi-core architectures" exposes a performance study of the particular case of the visual odometry (VO) problem using three different consumer desktop computers. Visual odometry is a special case of the Structure from Motion problem that can be effectively used in the Advanced Driver Assistant Systems (ADAS) context for autonomous driving. In this work, the VO solution is ported to a multicore framework for acceleration purposes, especially when using redundant spatiotemporal information and techniques such as FAST, BRIEF, Levenberg-Marqu$\operatorname{ardt/RANSAC}$ and a Kalman filter.

Nam and Hong present the paper entitled "Real-time abnormal situation detection based on particle advection in crowded scenes" that describes a method for the detection of abnormal situation in crowded scenes that operates in real time. The method is based on the computation of measures related to optical flow and similarity between direction histograms. The authors conducted experiments on four different datasets, obtaining promising results that demonstrates the effectiveness of the method.

The paper by Poiesi and Cavallaro entitled "Predicting and recognizing human interactions in public spaces" proposes a survey of methods for recognizing human interactions and propose a new method for predicting rendezvous areas (regions where people interact) using sparse motion information. The proposed method infers the direction of movement by calculating prediction lines from displacement vectors and temporally accumulates intersecting locations generated by prediction lines. These intersections are considered as candidates to be rendezvous areas and they are modeled by mixture of Gaussians.

Finally, Chun et al. in the work entitled "Real-time smart lighting control using human motion tracking from depth camera" focus on energy-efficient lighting systems as a contribution in the smart cities' energy field. The system architecture provides an automatic activity-dependent lighting environment as well as significant energy savings through the efficient integration of simplified computer vision strategies. Lighting control is based on estimated proximity to specific activity areas, target lighting areas, and heading direction of the individual.

We would like to acknowledge the authors for providing to this special issue their latest and most innovative research works. In addition, we would also like to thank the reviewers for helping us to prepare this high quality special issue. We are also really grateful to the Editors in Chief, Professors Nasser Kehtarnavaz and Matthias Carlsohn, for giving us the opportunity to guest-edit this special issue. We hope that the selected papers will contribute to enlighten the state of the art in the research concerning smart cities from a real-time computer vision perspective, including computer vision systems, hardware architectures, and algorithms. 